\title{
An immune tolerance approach using transient low-dose methotrexate in the ERT-naïve setting of patients treated with a therapeutic protein: experience in infantile-onset Pompe disease
}

\author{
Zoheb B. Kazi, MBBS ${ }^{1}$, Ankit K. Desai, MBBS ${ }^{1}$, R. Bradley Troxler, MD², David Kronn, MD, \\ Seymour Packman, MD ${ }^{4}$, Marta Sabbadini, PhD ${ }^{4}$, William B. Rizzo, MD ${ }^{5}$, Katalin Scherer, MD ${ }^{6}$, \\ Omar Abdul-Rahman, $\mathrm{MD}^{7}$, Pranoot Tanpaiboon, $\mathrm{MD}^{8}$, Sheela Nampoothiri, $\mathrm{MD}^{9}$, \\ Neerja Gupta, MD, DM ${ }^{10}$, Annette Feigenbaum, FRCP, DABMG ${ }^{11}$, Dmitriy M. Niyazov, MD ${ }^{12}$, \\ Langston Sherry, CNP ${ }^{12}$, Reeval Segel, $\mathrm{MD}^{13}$, Alison McVie-Wylie, PhD ${ }^{14}$, \\ Crystal Sung, PhD D(ABMLI) ${ }^{14}$, Alexandra M. Joseph, $\mathrm{PhD}^{14}$, Susan Richards, $\mathrm{PhD}^{14}$ and \\ Priya S. Kishnani, MD ${ }^{1}$
}

Purpose: To investigate immune tolerance induction with transient low-dose methotrexate (TLD-MTX) initiated with recombinant human acid a-glucosidase (rhGAA), in treatmentnaïve cross-reactive immunologic material (CRIM)-positive infantile-onset Pompe disease (IOPD) patients.

Methods: Newly diagnosed IOPD patients received subcutaneous or oral $0.4 \mathrm{mg} / \mathrm{kg}$ TLD-MTX for 3 cycles (3 doses/cycle) with the first 3 rhGAA infusions. Anti-rhGAA IgG titers, classified as highsustained (HSAT; $\geq 51,200, \geq 2$ times after 6 months), sustained intermediate (SIT; $\geq 12,800$ and $<51,200$ within 12 months), or low (LT; $\leq 6400$ within 12 months), were compared with those of 37 CRIM-positive IOPD historic comparators receiving rhGAA alone.

Results: Fourteen IOPD TLD-MTX recipients at the median age of 3.8 months (range, $0.7-13.5$ months) had a median last titer of 150 (range, $0-51,200$ ) at median rhGAA duration $\sim 83$ weeks (range, $36-122$ weeks). One IOPD patient (7.1\%) developed titers in the SIT range and one patient (7.1\%) developed titers in the HSAT range. Twelve of the 14 patients $(85.7 \%)$ that received TLD-MTX remained LT, versus 5/37 HSAT (peak 51,200-409,600), 7/37 SIT (12,800-51,000), and 23/37 LT (200-12,800) among comparators.

Conclusion: Results of TLD-MTX coinitiated with rhGAA are encouraging and merit a larger longitudinal study.

Genetics in Medicine (2019) 21:887-895; https://doi.org/10.1038/s41436018-0270-7

Keywords: alglucosidase alfa; antidrug antibodies; prophylactic immune tolerance induction; methotrexate; Pompe disease

\section{INTRODUCTION}

Pompe disease, also known as glycogen storage disease type II (OMIM 232300), is an autosomal recessive lysosomal storage disorder in which deficiency of the lysosomal enzyme acid $\alpha$ glucosidase (GAA, EC 3.2.1.20) results in a build-up of glycogen in cardiac, skeletal, and smooth muscle of affected individuals. ${ }^{1}$ Infantile-onset Pompe disease (IOPD) presents in the first days to weeks of life, with symptoms of hypotonia, cardiomyopathy, and respiratory insufficiency. Without treatment, death usually occurs before the age of 2 years. ${ }^{2-4}$

Alglucosidase alfa (recombinant human GAA; rhGAA) was approved for enzyme replacement therapy (ERT; this abbreviation denotes rhGAA use throughout this paper) in Pompe disease in 2006 (ref. ${ }^{5}$ ). Its administration has been

\footnotetext{
${ }^{1}$ Division of Medical Genetics, Department of Pediatrics, Duke University Health System, Durham, NC, USA; ${ }^{2}$ Department of Pediatrics, University of Alabama at Birmingham, Birmingham, AL, USA; ${ }^{3}$ Department of Pediatrics, New York Medical College, Valhalla, NY, USA; ${ }^{4}$ Division of Medical Genetics, Department of Pediatrics, University of California, San Francisco, CA, USA; ${ }^{5}$ Department of Pediatrics, University of Nebraska Medical Center, Nebraska Medical Center Omaha, Omaha, NE, USA; ${ }^{6}$ Department of Neurology, University of Arizona, Tucson, AZ, USA; ${ }^{7}$ Munroe-Meyer Institute for Genetics and Rehabilitation, University of Nebraska Medical Center, Omaha, NE, USA; ${ }^{8}$ Quest Diagnostics and Children's National Health System, Washington, DC, USA; ${ }^{9}$ Amrita Institute of Medical Sciences \& Research Centre, Kochi, Kerala, India; ${ }^{10}$ All India Institute of Medical Sciences, New Delhi, India; ${ }^{11}$ University of California, San Diego, CA, USA; ${ }^{12}$ Department of Pediatrics, Ochsner Health System, New Orleans, LA, USA; ${ }^{13}$ Medical Genetics Institute, Shaare Zedek Medical Center and the Hebrew University School of Medicine, Jerusalem, Israel; ${ }^{14}$ Sanofi Genzyme, Framingham, MA, USA. Correspondence: Priya S. Kishnani (priya.kishnani@duke.edu)

Z.B.K. and A.K.D. are co-first authors.
} 
shown to improve overall and ventilator-free survival in IOPD, with improved clinical outcomes. Today, many longterm survivors have reached adolescence. ${ }^{6-8}$ However, response to ERT is often affected by an immune response, which may increase infusion-associated reactions (IgE mediated), lead to mistargeting of delivered enzyme (IgG mediated), and/or reduce clinical efficacy (IgG mediated). ${ }^{9}$ Treatment and the immune response to ERT have been shown to be related to the patient's endogenous enzyme, commonly referred to as cross-reactive immunologic material (CRIM) status, with CRIM-negative patients developing high and sustained anti-rhGAA immunoglobulin G (IgG) antibody titers (HSAT; defined as titers of $\geq 51,200$ at $\geq 2$ time points at or beyond 6 months on rhGAA) and having a poor outcome in response to ERT compared with CRIM-positive patients. ${ }^{10}$ CRIM-negative patients cannot form the native enzyme and usually possess two severe GAA pathogenic variants; their immune systems recognize ERT as foreign and, as a result, form clinically important levels of anti-rhGAA IgG antibodies. ${ }^{10}$ CRIM-negative patients account for $\sim 25-32 \%$ of all patients with IOPD, ${ }^{11,12}$ and are likely to have a poor clinical outcome when treated with ERT alone. In contrast, CRIMpositive patients have some residual native enzyme, whether functional or nonfunctional, ${ }^{10}$ and are more likely to develop lower anti-rhGAA IgG titers or none. ${ }^{13}$

In a retrospective analysis by Banugaria et al., ${ }^{13} 39 \%(9 / 23)$ CRIM-positive IOPD patients treated with ERT had high anti-rhGAA IgG antibody titers, and their clinical outcomes were poor, similar to CRIM-negative patients; thus it was established that antibody status affects ERT response. At present, there are no predictive factors to determine which CRIM-positive patients will develop HSAT or sustained intermediate titers (SIT; defined as titers of $\geq 12,800$ and $<51,200$ within the first year on rhGAA).

Immune tolerance induction (ITI) protocols have been established as an approach to minimize the development of anti-rhGAA IgG antibodies and maintain low or absent antibody titers over time. Protocols for ITI using various immunomodulating drugs have been studied for prophylaxis to preempt immune response in ERT-naïve patients ${ }^{14-16}$ and for therapy to decrease existing anti-rhGAA antibodies in ERT-treated patients with already established immune responses. ${ }^{14,15,17,18}$ However, studies in the largest number of CRIM-negative patients naïve to ERT therapy have prophylactically combined rituximab and methotrexate (e.g., one course of rituximab $375 \mathrm{mg} / \mathrm{m}^{2}$ IV weekly four times; methotrexate $0.4 \mathrm{mg} / \mathrm{kg}$ subcutaneously every 2 weeks), with or without intravenous immunoglobulin, in efforts to preempt an immune response. ${ }^{14,15,17}$ In the past, there was no success in patients with an entrenched immune response and antibody titers persisted after multiple immune modulating treatment regimens. ${ }^{19,20}$ Addition of the plasma cell-targeting agent bortezomib ${ }^{21}$ or other therapies in patients with established HSAT has proven successful in minimizing antibody titers, yet required prolonged immunosuppression arising from the use of maintenance doses of rituximab and methotrexate along with bortezomib. ${ }^{18,22}$

A short course of prophylactic ITI in CRIM-negative IOPD patients has improved clinical outcomes as compared with ERT monotherapy by preempting the development of HSAT and thus preventing the consequent loss of ERT efficacy. As demonstrated in a prior study by Banugaria et al., ${ }^{23}$ using a three-drug ITI regimen (methotrexate, rituximab, and intravenous immunoglobulin) initiated concurrently with ERT in 7 CRIM-negative classic IOPD patients, four patients never seroconverted (developed antibodies), one patient died of respiratory failure, and two patients required additional ITI courses, which left their antibody titers lower than in ERTtreated CRIM-negative infants without ITI. A follow-up study with the same three-drug ITI regimen in 19 CRIM-negative patients, including the 7 patients in Banugaria et al., ${ }^{23}$ showed that 15 of 19 patients either did not seroconvert or maintained low antibody titers, in contrast to the natural course of CRIMnegative patients on ERT monotherapy. Only 1 of these 19 patients broke tolerance and developed HSAT. This patient was subsequently rescued using a bortezomib-based ITI protocol. $^{24}$ The same prophylactic ITI regimen successfully induced tolerance in the CRIM-positive younger sibling of a CRIM-positive Pompe patient who had developed HSAT on ERT monotherapy. ${ }^{25}$ Prophylactic ITI protocols concurrent with ERT initiation are used increasingly by treating physicians worldwide and are considered a standard of care for CRIM-negative patients.

A subset of CRIM-positive patients also develop a sustained immune response, ${ }^{13}$ and it is difficult to predict which CRIMpositive individuals will develop a transient antibody response (seroconvert) or go on to develop HSAT or SIT. Anti-rhGAA antibody titers $\geq 12,800$ were associated with an increase in ERT clearance in a pharmacokinetic study reported in the alglucosidase alfa prescribing information. ${ }^{26}$ Clinical response to ERT will likely be better conserved by preempting HSAT/ SIT than by striving to reduce it once it occurs. ${ }^{25}$ Indeed, as shown in our studies, once antibodies develop to a significant titer, the intensity of immune suppression to establish tolerance is much greater than that in prophylactic regimens. Moreover, the potential for loss of viable muscle tissue due to loss of ERT activity due to such high titered immune responses is a critical consideration. Thus every effort to prevent the development of these deleterious antibodies is important and study of prophylactic ITI regimens at ERT initiation merits extension into CRIM-positive patients, given that a large subset of them develop significant antibodies likely to have deleterious clinical impact.

Preclinically, a transient low dose of methotrexate alone coinitiated with ERT sustainably reduced anti-rhGAA IgG response in a GAA knockout mouse model of Pompe disease. ${ }^{27}$ Studies in wild-type and GAA knockout mice suggest a mechanism for methotrexate ITI that involves an IL10 and $\mathrm{B}$ regulatory cell-dependent mechanism. ${ }^{28,29}$ Other immune tolerance-inducing mechanisms may also come into play. In light of these results, and given that methotrexate has 

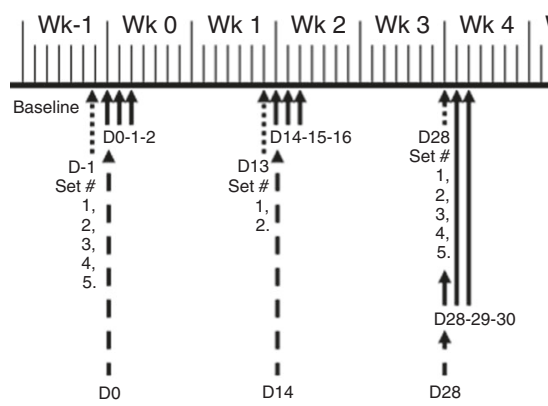

$\begin{array}{rr}\mathbf{4} & \mathbf{1} \\ \mathrm{D} 42 & \mathrm{D} 5 \\ \text { Set \# } & \text { Set } \\ 1, & 2 \\ 2 . & 3 \\ \mathbf{I} & 4 \\ \mathbf{I} & 5 \\ \mathbf{I} & \\ \mathbf{I} & \\ \mathbf{I} & \\ \text { D42 } & \end{array}$

$\mathbf{4}$
D56
Set \#
1,
2,
3,
4,
5.
$\mathbf{4}$
$\mathbf{I}$
$\mathbf{I}$
$\mathbf{1}$
D56

I
I
I
I
I
I
I
D70

$\begin{array}{rrr}\mathbf{4} & \mathbf{I} & \mathbf{1} \\ \mathrm{D} 84 & \mathbf{I} & \mathrm{D} 112 \\ \text { Set \# } & \mathbf{I} & \text { Set \# } \\ 1, & \mathbf{I} & 3, \\ 2, & \mathbf{I} & 4, \\ 3, & \mathbf{I} & 5 . \\ 4, & \mathbf{I} & \mathbf{1} \\ 5 . & \mathbf{I} & \mathbf{I} \\ \mathbf{4} & \mathbf{I} & \mathbf{I} \\ \mathbf{I} & \mathbf{I} & \mathbf{I} \\ \mathbf{I} & \mathrm{D} 98 & \mathbf{I} \\ \mathbf{I} & & \mathrm{D} 112 \\ \text { D84 } & & \end{array}$

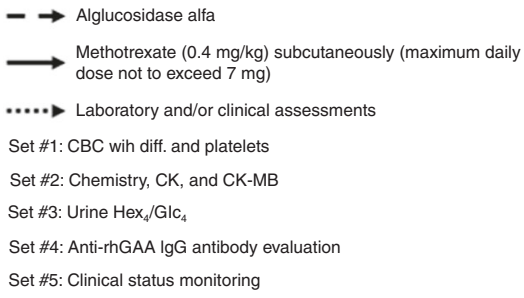

Fig. 1 Study timeline for infantile Pompe disease patients receiving enzyme replacement therapy (ERT) infusions every other week. $C B C$ with diff. complete blood count with differential, $C K$ creatinine kinase, $H e x_{4} / G / c_{4}$ glucose tetrasaccharide, $I g G$ immunoglobulin $G$, rhGAA recombinant human acid alpha glucosidase

a favorable safety profile in the doses proposed, is inexpensive and widely accessible, and is administered only during the initial phases of therapy, we undertook the first human protocol incorporating transient use of methotrexate at the initiation phase of ERT in naïve patients with IOPD. For ITI, we investigated a transient, low dose of methotrexate administered only during the first three ERT infusions and then stopped (Fig. 1). This is referred to as the transient lowdose methotrexate (TLD-MTX) protocol. We hypothesized that CRIM-positive patients with Pompe disease who receive methotrexate in this manner in the naive setting will not seroconvert or will have a blunted immune response in comparison with the CRIM-positive patients treated with ERT monotherapy.

\section{MATERIALS AND METHODS \\ Patients and inclusion and exclusion criteria}

Patients with a confirmed diagnosis of IOPD (two confirmed GAA pathogenic variants) and a low GAA enzyme activity (in blood, muscle, or skin) who were CRIM-positive and ERTnaïve were enrolled in the study. CRIM status was determined by western blot analysis and confirmed by GAA genotype or was predicted from GAA genotype alone. ${ }^{11}$ IOPD was defined as symptom onset at $\leq 12$ months of age with cardiomyopathy.

Upon a new diagnosis of Pompe disease, the detailed protocol was shared with the patient's local treating physician, including the dosing schedule of methotrexate, IgG antibody monitoring guidelines, and laboratory analyses for safety measures. Follow-up data from the local treating team were requested every 3 months. Data collection was completed 16 June 2017 or when at least 6 months of follow-up data were available. If a patient broke tolerance their latest immune responses beyond 16 June 2017 have been presented.
Previously described methods were used for determining CRIM status, ${ }^{11,12}$ GAA pathogenic variants, ${ }^{7}$ and anti-rhGAA IgG antibody titers. ${ }^{7}$ Clinical outcomes used were overall survival and ventilator-free survival for patients who received TLD-MTX protocol.

\section{Ethical approval and parent, guardian, or adult patient consent}

All patients were enrolled in an Institutional Review Board (IRB)-approved study. Thirteen patients were enrolled in a Duke University IRB-approved protocol 00001562 (LDN6709 Site 206; ClinicalTrials.gov NCT01665326: Determination of Cross-Reactive Immunological Material [CRIM] Status and Longitudinal Follow-up of Individuals with Pompe disease). One patient was enrolled in an IRB-approved study at an institution local to the patient (Shaare Zedek Medical Center, Jerusalem, Israel). Informed consent was obtained in writing from parent(s) or legal guardian(s) of minor patients for their respective studies.

\section{Study design}

The study timeline for ERT and methotrexate dosing is shown in Fig. 1. Patients received intravenous (IV) infusions of ERT at $20 \mathrm{mg} / \mathrm{kg}$ body weight every other week (EOW) based on the package insert or on a different regimen based on decision of the treating clinician.

Methotrexate at $0.4 \mathrm{mg} / \mathrm{kg}$ body weight was administered subcutaneously (or orally if subcutaneous administration was not possible) on three consecutive days/MTX cycle/infusion; with the first three ERT infusions (i.e., on days 0,14 , and 28 for patients infused EOW) for a total of 3 cycles. On the day of ERT infusion, methotrexate was administered 15 minutes (if subcutaneous) or 1 hour (if oral) before infusion initiation 
and again on the following 2 days. To exemplify the schedule, in a patient receiving ERT infusion EOW, methotrexate was administered on days 0,1 , and 2 (first cycle); days 14, 15, and 16 (second cycle); and days 28, 29, and 30 (third cycle). As stated earlier, the dose and frequency of ERT could be increased at the discretion of the treating physician, based on patients' clinical status, in which case methotrexate cycles were adjusted accordingly (see Fig. S1 for details).

The methotrexate dose was stipulated to be withheld if the absolute neutrophil count (ANC) was $<750 / \mathrm{mm}^{3}$ or liver function tests (aspartate aminotransferase [AST] and alanine aminotransferase [ALT]) were $>3$ times their respective baseline values. Folinic acid supplementation, although not directed in the protocol, could be given at the treating physician's discretion if there was a concern with methotrexate toxicity.

\section{Immune response}

Anti-rhGAA IgG antibodies were determined by Sanofi Genzyme (Framingham, MA, USA) using enzyme-linked immunosorbent assays and confirmed using radioimmunoprecipitation as described previously. ${ }^{7}$ Antibody analyses were recommended to be performed at days $-1,28,56,84,112$, $140,168,196,224,252,280,308,336$, and 364 .

\section{Hematologic and biochemical analyses}

On days -1 (baseline), 13, 28, 42, 56, 84, 168, 252, 336, and 364 , blood samples were recommended to be collected for hematologic and biochemical analyses for patients receiving ERT EOW. The sampling schedule was modified for patients receiving ERT weekly (Fig. S1). Complete and differential blood counts and platelets were evaluated and monitored for platelet counts $<50,000 / \mathrm{mm}^{3}$, ANC $<750 / \mathrm{mm}^{3}$, and treatment-resistant infections. Biochemical analyses included ALT, AST, creatine kinase (CK), and CK-MB levels. Posttreatment increases in AST and ALT levels were noted if exceeding three times their respective baseline levels.

\section{Statistical analysis}

Patients were classified into HSAT ( $\geq 51,200$ on $\geq 2$ occasions at or beyond 6 months on ERT $\left.{ }^{13,22}\right)$, SIT $(\geq 12,800$ and $<51,200$ within the first year of ERT), and low titer (LT; $\leq 6400$ within the first year of ERT) groups based on the longitudinal anti-rhGAA IgG titers. ${ }^{23}$ The lower bound of SIT, $\geq 12,800$, was associated with increase in ERT clearance in a pharmacokinetic study reported in the alglucosidase alfa prescribing information; the upper bound of LT, $\leq 6400$, was 1 dilution level below this. Medians and ranges were determined for baseline, peak, and last titers, although our focus was on longitudinal titers through time because sustained titers affect clinical response. ${ }^{30}$

\section{Comparator group for CRIM-positive IOPD}

A retrospective chart review of 37 CRIM-positive patients with IOPD was conducted from the original rhGAA clinical trials, including patients on ERT for $\geq 6$ months who did not receive immunomodulation. ${ }^{6,7,10,31,32}$ The GAA variant data and age at ERT initiation were compared with our cohort of TLD-MTX recipients. Immune responses over time were classified into HSAT, SIT, and LT as defined earlier, and time to seroconversion, peak titers, titers at weeks 12,24 , and 52 were compared with our present cohort of TLD-MTX recipients.

\section{RESULTS}

\section{Patient disposition}

Fourteen ERT-naïve patients with Pompe disease received TLD-MTX protocol at the median age of 3.8 months (range, 0.7-13.5 months). Longitudinal follow-up data of $>6$ months were available for 14 patients with IOPD (13 CRIM-positive patients and 1 CRIM-negative patient). Of the 28 total GAA variants in the TLD-MTX group, 18 (64.3\%), 3 (10.7\%), 3 (10.7\%), $3(10.7 \%)$, and 1 (3.6\%) were missense, frameshift, initiator codon, nonsense, and splice-site variants, respectively. The patients' baseline demographics are presented in Table 1. The CRIM-negative patient (IOPD9) who received TLD-MTX protocol is an international patient initially thought to be CRIM-positive. No patients in this cohort were diagnosed via newborn screening.

\section{ERT monotherapy-treated CRIM-positive patients (comparator group)}

Thirty-seven CRIM-positive patients were identified who received ERT monotherapy at a median age of 6.9 months (range, 0.5-43.1 months). Age at ERT start was later in the ERT monotherapy group (median, 6.9 months) as compared with the TLD-MTX group (median, 3.8 months). Of the 74 total GAA variants in the ERT monotherapy group, 47 (63.5\%), 6 (8.1\%), 1 (1.4\%), 7 (9.5\%), 2 (2.7\%), 7 (9.5\%), and 4 (5.4\%) were missense, frameshift, initiator codon, nonsense, splice-site, unknown, and in-frame deletion variants, respectively. The GAA variant data in the CRIM-positive patients in the comparator group (ERT monotherapy) were similar to those in the TLD-MTX group (Fig. S3).

Five (13.5\%), 7 (18.9\%), and $25(67.6 \%)$ of the 37 IOPD monotherapy patients developed HSAT, SIT, and LT, respectively; thus, $32.4 \%$ developed SIT or HSAT overall. All HSAT patients seroconverted by 4 weeks; for the SIT and LT patients, median time to seroconversion was 4 weeks ( $4-8$ weeks, $n=7$ ) and 8 weeks ( $4-64$ weeks, $n=$ 23), respectively; 2 patients did not seroconvert. Group median peak titers were 204,800 (range, 51,200-409,600) for HSAT (median time of peak, week 82), 25,600 (range, 12,800-51,200) for SIT (week 12), and 800 (range, 200-12,800) for LT (week 38). Group median last titers (at group median times on ERT) were 102,400 for HSAT (week 94) (range, 51,200-409,600), 1600 for SIT (week 104) (range, 200-25,600), and 400 for LT (week 130) (range, 0-12,800); individual data are shown in Fig. 2. These data were used for comparison with the present TLD-MTX-treated IOPD patients (Fig. 2 and Fig. S2). Comparison of median anti-rhGAA IgG antibody titers at 
Table 1 Baseline demographic characteristics of IOPD patients that received TLD-MTX protocol

\begin{tabular}{|c|c|c|c|c|c|c|c|}
\hline Patient & Sex & \multicolumn{2}{|c|}{ GAA pathogenic variants } & $\begin{array}{l}\text { Age at start } \\
\text { of ERT }\end{array}$ & $\begin{array}{l}\text { ERT dose } \\
\text { and } \\
\text { frequency }^{\mathrm{a}}\end{array}$ & $\begin{array}{l}\text { Route of MTX } \\
\text { administration }\end{array}$ & TLD-MTX protocol deviation \\
\hline IOPD2 & M & $\begin{array}{l}\text { c. } 1004 \mathrm{G}>\mathrm{A} \\
\text { p.Gly335Glu }\end{array}$ & $\begin{array}{l}\text { c. } 1841 C>A \\
\text { p.Thr614Lys }\end{array}$ & 3.3 months & $\begin{array}{l}20 \mathrm{mg} / \mathrm{kg} \\
\mathrm{EOW}\end{array}$ & NA & None \\
\hline IOPD4 & $\mathrm{F}$ & $\begin{array}{l}\text { c. } 2560 C>T \\
\text { p.Arg854X }\end{array}$ & $\begin{array}{l}\text { c. } 1466 \mathrm{~A}>\mathrm{G} \\
\text { p.Asp489Gly }\end{array}$ & 0.8 months & $\begin{array}{l}20 \mathrm{mg} / \mathrm{kg} \\
\text { EOW }\end{array}$ & MTX administered SC & $\begin{array}{l}\text { 3rd cycle of MTX \& ERT was } \\
\text { administered on week } 5 \text { instead } \\
\text { of week } 4\end{array}$ \\
\hline IOPD6 & $\mathrm{F}$ & $\begin{array}{l}\text { c. } 1114 C>T \\
\text { p. His372Tyr }\end{array}$ & $\begin{array}{l}\text { c. } 1979 \mathrm{G}>\mathrm{A} \\
\text { p.Arg660His }\end{array}$ & 11.2 months & $\begin{array}{l}20 \mathrm{mg} / \mathrm{kg} \\
\mathrm{EOW}\end{array}$ & MTX administered PO & None \\
\hline IOPD7 & $\mathrm{F}$ & $\begin{array}{l}\text { c.2456G>C } \\
\text { p.Arg819Pro }\end{array}$ & $\begin{array}{l}\text { c. } 2456 \mathrm{G}>\mathrm{C} \\
\text { p.Arg819Pro }\end{array}$ & 1.2 months & $\begin{array}{l}20 \mathrm{mg} / \mathrm{kg} \\
\mathrm{EOW}\end{array}$ & MTX administered SC & None \\
\hline IOPD8 & $\mathrm{F}$ & $\begin{array}{l}\text { c. } 525 \text { delT } \\
\text { p. } \\
\text { Glu176Argfs } * 45\end{array}$ & $\begin{array}{l}\text { c. } 2297 \mathrm{~A}>C \\
\text { p.Tyr766Ser }\end{array}$ & 4.6 months & $\begin{array}{l}20 \mathrm{mg} / \mathrm{kg} \\
\text { EOW }\end{array}$ & MTX administered SC & $\begin{array}{l}\text { 3rd cycle of MTX \& ERT was } \\
\text { administered on week } 5 \text { instead } \\
\text { of week } 4\end{array}$ \\
\hline IOPD9 $^{b}$ & $\mathrm{~F}$ & $\begin{array}{l}\text { c. } 1 \mathrm{~A}>\mathrm{G} \\
\text { Initiator codon }\end{array}$ & $\begin{array}{l}\text { c. } 1 \mathrm{~A}>\mathrm{G} \\
\text { Initiator codon }\end{array}$ & 12.9 months & $\begin{array}{l}20 \mathrm{mg} / \mathrm{kg} \\
\mathrm{EOW}\end{array}$ & MTX administered SC & None \\
\hline IOPD10 & $\mathrm{F}$ & $\begin{array}{l}\text { c. } 1942 \mathrm{G}>\mathrm{A} \\
\text { p.Gly648Ser }\end{array}$ & $\begin{array}{l}\text { c. } 1942 \mathrm{G}>\mathrm{A} \\
\text { p.Gly648Ser }\end{array}$ & 1.7 months & $\begin{array}{l}20 \mathrm{mg} / \mathrm{kg} \\
\mathrm{EOW}\end{array}$ & MTX administered SC & $\begin{array}{l}\text { 3rd dose of 3rd cycle of MTX was } \\
\text { skipped }\end{array}$ \\
\hline IOPD13 & $\mathrm{F}$ & $\begin{array}{l}\text { c. } 1979 \mathrm{G}>\mathrm{A} \\
\text { p.Arg660His }\end{array}$ & $\begin{array}{l}\text { c. } 2560 C>T \\
\text { p.Arg } 854 X\end{array}$ & 4.5 months & $\begin{array}{l}20 \mathrm{mg} / \mathrm{kg} \\
\mathrm{EOW}^{\mathrm{c}}\end{array}$ & MTX administered SC & None \\
\hline IOPD14 & $\mathrm{F}$ & $\begin{array}{l}\text { c.525delT } \\
\text { p. } \\
\text { Glu176Argfs*45 }\end{array}$ & $\begin{array}{l}\text { c. } 1979 \mathrm{G}>\mathrm{A} \\
\text { p.Arg } 660 \mathrm{His}\end{array}$ & 4.0 months & $\begin{array}{l}20 \mathrm{mg} / \mathrm{kg} \\
\mathrm{EOW}\end{array}$ & MTX administered PO & $\begin{array}{l}\text { 1st MTX dose of 3rd cycle was } \\
\text { skipped }\end{array}$ \\
\hline
\end{tabular}

ANC absolute neutrophil count, CRIM cross-reactive immunologic material, EOW every other week, ERT enzyme replacement therapy, IOPD infantile-onset Pompe disease, PO orally (per os), SC subcutaneous, TLD-MTX transient low-dose methotrexate

${ }^{a} E R T$ dose and frequency were determined based on the clinical judgment of the treating physician

bPatient IOPD9 is CRIM-negative

'ERT dose was changed to $40 \mathrm{mg} / \mathrm{kg} /$ weekly after two infusions

weeks 12,24 , and 52 , and median peak titers is shown in Table 2.

\section{Immune response: transient low-dose methotrexate recipients}

The immune response for each TLD-MTX treated patient over time is shown in Fig. 2, and summarized in Table 2 and Table 3. The median last titer was 150 (range, 0-51,200) at median time on ERT of 83 weeks (range, 36-122 weeks). One IOPD patient (IOPD14; 7.1\%) developed titers in the SIT range and one patient (IOPD13; 7.1\%) developed titers in the HSAT range. Patient IOPD13 had a titer of 102,400 at weeks 76 and 81 decreasing to 51,200 at week 85. Patient IOPD14 had a titer of 25,600 at week 28 only, and maintained titers of 12,800 at weeks 12,16 , and 24, and 32 decreasing to 6400 at week 36. These 2 TLD-MTX recipient patients (IOPD13 and IOPD14) had the highest titers observed in the study; the other 12 TLD-MTX recipients remained LT. One patient (IOPD2) had titers remaining $<100$ (seropositive on screening assay, but below the limit of titer measurement). 


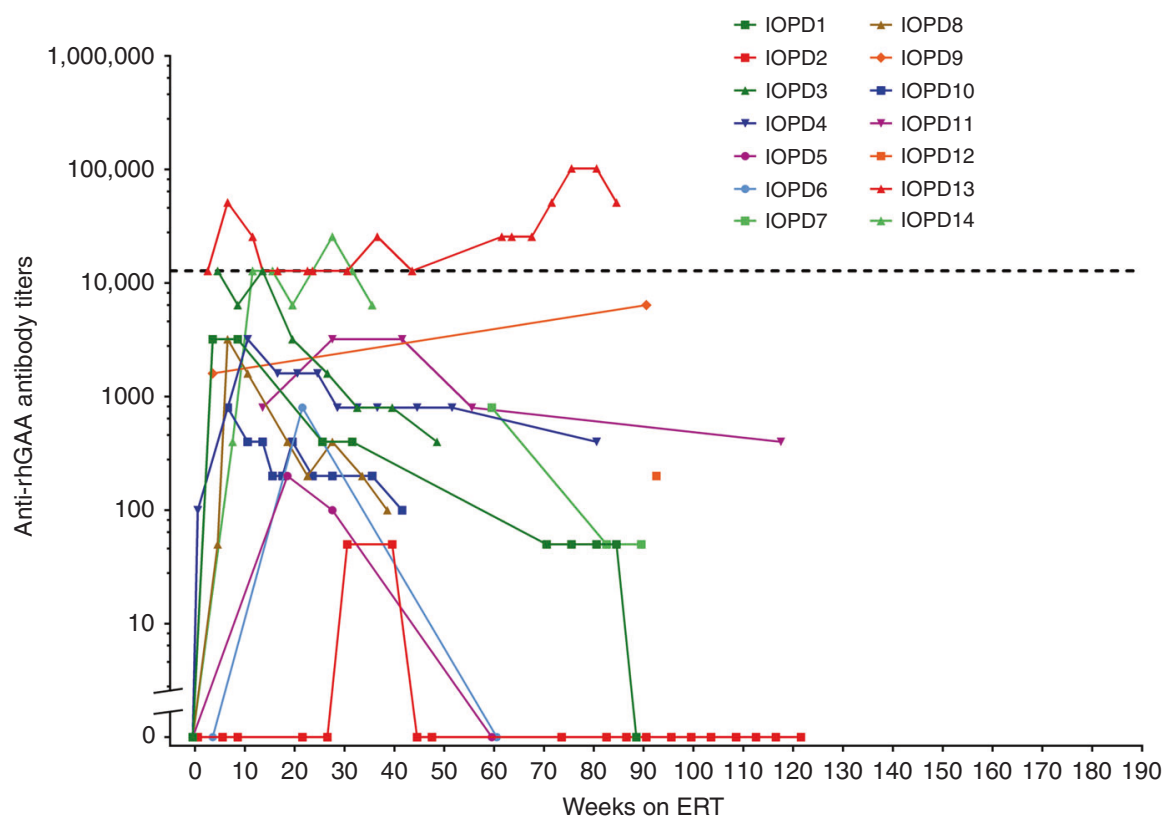

Fig. 2 Immune response over time in the current TLD-MTX-treated CRIM-positive IOPD patients. Each patient's trajectory is graphed individually as a curve. Positive titers $<100$ are shown as titers of 50. CRIM cross-reactive immunological material, ERT enzyme replacement therapy, IOPD infantile-onset Pompe disease, rhGAA recombinant human acid alpha glucosidase, TLD-MTX transient low-dose methotrexate

\section{Safety}

Hematologic and biochemical analyses

ANC, AST, and ALT data in the first 6 weeks on ERT were available for all patients except IOPD7 (missing all 3 measures), and IOPD2 and IOPD13 (missing baseline AST and ALT). Patients IOPD1 and IOPD3 developed ANC $<750$ cells $/ \mathrm{mm}^{3}$. Neutropenia in these two patients was transient, and ANC returned to normal levels. Patient IOPD5 developed increases exceeding three times baseline levels of both AST and ALT; patients IOPD3 and IOPD6 had such increases only in ALT. Infection incidence during the time of TLD-MTX administration was available on nine patients. Two patients

Table 2 Comparison of longitudinal immune response between ERT monotherapy and TLD-MTX groups ${ }^{\mathrm{a}}$ CRIM-positive IOPD on ERT monotherapy $(n=37)$

\begin{tabular}{|c|c|c|c|}
\hline Groups & HSAT & SIT & LT \\
\hline Percent & $13.5 \%(n=5)$ & $18.9 \%(n=7)$ & $67.6 \%(n=25)$ \\
\hline Median titers (week 12) & $25,600$ (range: $12,800-51,200)$ & 12,800 (range: $6400-25,600$ ) & 400 (range: 0-6400) \\
\hline Median titers (week 24) & 25,600 (range: 6400-204,800) & 12,800 (range: $6400-51,200$ ) & 400 (range: 0-3200) \\
\hline Median titers (week 52) & 51,200 (range: $12,800-102,400$ ) & 3200 (range: 400-12,800) & 200 (range: 0-6400) \\
\hline Median peak titers & 204,800 (range: $51,200-409,600$ ) & 25,600 (range: $12,800-51,200$ ) & 800 (range: 0-12,800) \\
\hline Time since ERT initiation & 82 weeks (range: $24-130)$ & 12 weeks (range: 8-24) & 38 weeks (range: 8-172) \\
\hline Groups & HSAT & SIT & LT \\
\hline Percent & $7.1 \%(n=1)$ & $7.1 \%(n=1)$ & $85.7 \%(n=12)^{\mathrm{b}}$ \\
\hline Median titers (week 12) & IOPD13: 12,800 & IOPD14: 25,600 & 1600 (range: 0-12,800) \\
\hline Median titers (week 24) & IOPD13: 12,800 & IOPD14: 12,800 & 600 (range: 0-3200) \\
\hline Median titers (week 52) & IOPD13: 12,800 & NA & $250^{c}$ (range: $0-800$ ) \\
\hline
\end{tabular}

CRIM cross-reactive immunological material, ERT enzyme replacement therapy, HSAT high and sustained antibody titers, IOPD infantile-onset Pompe disease, LT low titers, NA not applicable, SIT sustained intermediate titers, TLD-MTX transient low-dose methotrexate

${ }^{a}$ All patients in ERT + TLD-MTX group and 35/37 patients in ERT monotherapy group seroconverted

bone patient had peak titers of 12,800 twice (week 5 and week 12) on ERT but maintained low titers throughout, so was included in the LT group

'Patients who tested seropositive but had titers $<100$ were deemed to have a titer of 50 
Table 3 Immune response for IOPD patients that received TLD-MTX protocol with > 6 months of follow-up data available

\begin{tabular}{lllll} 
Patient & $\begin{array}{l}\text { Peak } \\
\text { antibody } \\
\text { titer }\end{array}$ & $\begin{array}{l}\text { Time on ERT } \\
\text { (peak titer) } \\
\text { (weeks) }\end{array}$ & $\begin{array}{l}\text { Last } \\
\text { antibody } \\
\text { titer }\end{array}$ & $\begin{array}{l}\text { Time on ERT } \\
\text { (last titer) } \\
\text { (weeks) }\end{array}$ \\
\hline IOPD1 & 3200 & 4 & 0 & 89 \\
IOPD2 & $<100$ & 31 & 0 & 122 \\
IOPD3 & $12,800^{\text {a }}$ & 5 & 400 & 49 \\
IOPD4 & 3200 & 11 & 400 & 81 \\
IOPD5 & 200 & 19 & 0 & 60 \\
IOPD6 & 800 & 22 & 0 & 61 \\
IOPD7 & 800 & 60 & $<100$ & 90 \\
IOPD8 & 3200 & 7 & 100 & 39 \\
IOPD9 & 6400 & 91 & 6400 & 91 \\
IOPD10 & 800 & 7 & 100 & 42 \\
IOPD11 & 3200 & 28 & 400 & 118 \\
IOPD12 & 200 & 93 & 200 & 93 \\
IOPD13 & $102,400^{\mathrm{b}}$ & 76 & 51,200 & 85 \\
IOPD14 & $25,600^{\mathrm{c}}$ & 28 & 6400 & 36 \\
Median & $\mathbf{3 2 0 0}$ & $\mathbf{2 5}$ & $\mathbf{1 5 0}$ & $\mathbf{8 3}$ \\
\hline
\end{tabular}

EOW every other week, ERT enzyme replacement therapy, IOPD infantile-onset Pompe disease, TLD-MTX transient low-dose methotrexate

aPatient IOPD3 only received a single dose of methotrexate in cycles 2 and 3 instead of 3 doses

${ }^{b}$ Patient IOPD13 had an increase in dose of ERT from $20 \mathrm{mg} / \mathrm{kg} / \mathrm{EOW}$ to $40 \mathrm{mg} /$ $\mathrm{kg} / \mathrm{EOW}$ at week 4 on ERT

'Patient IOPD14 had skipped a dose of methotrexate on cycle 3

had hospitalizations related to infections around the time of methotrexate administration. One patient (IOPD8) was hospitalized for rhinovirus infection and the other patient (IOPD13) was hospitalized for concerns of underlying respiratory infections. No infections were noted in the remaining seven patients (IOPD1, IOPD5, IOPD7, IOPD9, IOPD10, IOPD12, and IOPD14).

\section{Clinical outcome}

Overall and ventilator-free survival

At baseline, four patients (IOPD2, IOPD3, IOPD5, and IOPD10) were invasively ventilated, two patients (IOPD8 and IOPD13) needed bilevel positive airway pressure (BiPAP) support, one patient (IOPD14) needed high-flow nasal cannula support, and seven patients required no support. Two of these 14 patients, IOPD3 and IOPD14, died at the age of 20 months and 15 months, respectively. The cause of death was cardiorespiratory failure secondary to the progression of disease in IOPD3 and respiratory arrest secondary to viral infection during the flu season for IOPD14. For the remaining 12 surviving patients only 1 patient (IOPD2) needed ventilator support, which was only needed during sleep. The remaining 11 patients do not need any support. The median age for the 12 surviving patients was 4.1 years (range: $2.3-5.7$ years).

\section{DISCUSSION}

We have developed an ITI protocol using TLD-MTX, a brief course (a total of 9 doses) of methotrexate in patients with CRIM-positive IOPD, which may also be considered for CRIM-negative IOPD patients lacking access to rituximab. We demonstrate the feasibility of implementation of this protocol in 13 centers in the United States, Israel, and India, a collaboration that demonstrates how physicians can work together worldwide to leverage clinical learning in rare diseases and in this case, mitigate the deleterious impact of immune response on ERT therapy. Brief-course, low-dose, single-drug methotrexate administered concurrently with rhGAA initiation in treatment-naïve patients with Pompe disease resulted in the cohort's median last titer of 150 (range, 0-51,200) at median time of 83 weeks (range, 36-122 weeks) of ERT. One patient (IOPD13) had antibodies in the HSAT range and one patient (IOPD14) had antibodies in the SIT range. All other methotrexate recipients remained LT throughout, unlike the responses in the comparator group. IOPD patients in the comparator group had a similar GAA variant profile. Importantly, all patients in this study were identified clinically and thus were symptomatic at time of diagnosis, again making the two groups very comparable. We collected data on overall and invasive ventilator-free survival on TLD-MTX recipient patients. Two of these fourteen patients (IOPD3 and IOPD14) were deceased and only one patient (IOPD2) needed ventilator support at night. The main focus of this study was ameliorating the deleterious antibody response to rhGAA. The deleterious effect of antibody titers in HSAT and SIT on cardiac and motor response has been previously published. ${ }^{10,13}$

Two patients (IOPD13 and IOPD14) developed antibodies in the SIT/HSAT range. Patient IOPD13 had an increase in dose of ERT from $20 \mathrm{mg} / \mathrm{kg} / \mathrm{EOW}$ to $40 \mathrm{mg} / \mathrm{kg} / \mathrm{EOW}$ at week 4 on ERT. The bortezomib-based protocol was recommended for this patient with HSAT, but the parents declined. Importantly, patient IOPD14, whose last titer was 6400 (week 36 ), had skipped a dose of methotrexate on cycle 3.

It is important to note that $32.4 \%$ of patients in the comparator group developed HSAT or SIT versus $14.2 \%$ of patients in TLD-MTX group. It appears that, based on expectations from historical data, TLD-MTX possibly blunted the immune response overall as demonstrated by the finding that $86.7 \%$ of patients in our cohort maintained low titers. Our patient cohort was younger, which could be more indicative of severe disease or earlier diagnosis. None of the patients were identified via newborn screening and all were clinically identified through clinical features of infantile Pompe disease including cardiomyopathy.

One of the patients was CRIM-negative, suggesting that TLD-MTX can be attempted in CRIM-negative patients in parts of the world where ITI with rituximab is not feasible. A study on a larger CRIM-negative cohort would be needed to establish the efficacy of TLD-MTX in that population for this purpose. 
No serious adverse events (AEs) were related to methotrexate. Some patients had methotrexate doses postponed because of their clinical status. Neutropenia (ANC $<750$ cells/ $\mathrm{mm}^{3}$ ) affected 2 of 13 IOPD patients with ANC data (15.4\%; IOPD1 and IOPD3); this was transient, and ANC returned to normal levels. Two of nine patients had hospitalizations related to infections around the time of methotrexate administration with complete resolution. Overall, there was a reasonably good tolerance to the methotrexate protocol in these fragile patients.

Our protocol uses only one-fourth to one-seventh of the methotrexate dose typical for cancer chemotherapy, and its brief time course avoids prolonged immunosuppression. Methotrexate is inexpensive and has a wide geographic availability; its lack of B-cell suppression may confer an advantageous safety profile in CRIM-positive patients as opposed to combination regimens including rituximab. ${ }^{15,23}$ TLD-MTX is also less expensive than regimens including biologics or lengthy immunosuppression.

Our data show the importance of 3 cycles of methotrexate for clinical efficacy. While 1-cycle methotrexate has not been studied systematically for ITI in humans, our patient who received less than 3-cycle seemed to have a higher immune response than those who followed the 3-cycle protocol. Our study did not formally compare 1 and 3 cycles (which had similar preclinical results); nonetheless, we hypothesize that 3 cycles are beneficial at this low dose to maintain B-cell regulation, without suppressing immunity altogether, as would be expected of high-dose methotrexate.

This report of clinical ITI experience using single-drug methotrexate is confined to CRIM-positive patients, except for IOPD9, who was predicted to be CRIM-negative based on $G A A$ variants. Further study is needed to determine potential applicability of this methotrexate protocol in a larger cohort of CRIM-positive patients and in CRIM-negative patients in regions where rituximab is not available. The TLD-MTX protocol requires specific evaluation before being applied in CRIM-negative patients, the highest-risk group; any empirical clinical use that is attempted will provide valuable experiential data. Further study is warranted in a larger cohort of IOPD patients, and should also evaluate longer-term outcomes such as overall survival, ventilator-free survival, reduction in left ventricular mass index, and urinary glucose tetrasaccharide $\left(\mathrm{Glc}_{4}\right)$.

Preclinical findings ${ }^{33}$ suggest that with further study, TLDMTX, which is given as a brief course at enzyme initiation (unlike ITI regimens requiring readministration over the course of treatment), may be appropriate for prevention of immune responses in other diseases treated by therapeutic enzymes. Other incipient immunomodulation methods are also being studied, such as anti-CD4 monoclonal antibodies, ${ }^{34}$ Tregitopes (peptides stimulating regulatory $\mathrm{T}$-cell expansion and activation), ${ }^{35}$ rapamycin, ${ }^{16}$ or tolerogenic nanoparticles containing rapamycin. ${ }^{36}$ Antidrug antibody responses and the approaches needed to preempt them may differ among diseases and therapeutic proteins. Further study is needed to understand the mechanism of methotrexate effects, evaluate safety and efficacy in larger CRIM-positive and CRIMnegative cohorts, and monitor long-term outcomes of TLDMTX-only ITI in Pompe disease.

\section{ELECTRONIC SUPPLEMENTARY MATERIAL}

The online version of this article (https://doi.org/10.1038/s41436018-0270-7) contains supplementary material, which is available to authorized users.

\section{ACKNOWLEDGEMENTS}

This study was supported in part by Sanofi Genzyme and the Lysosomal Disease Network (U54NS065768). Karen Welch and Christopher Abhulime of Sanofi Genzyme contributed laboratory antibody expertise and maintained validation of the assays used. Rick Fang of Sanofi Genzyme provided pharmacokinetic and pharmacodynamic modeling. Contributing caregivers Stephanie Dearmey, Einat Zivi, Dhanya Yesodharan, Kathleen Crosby, Jennifer Goodwin, Jennifer Cook, Matthew Stein, Valerie Marrero-Stein, and the late James Weisfeld-Adams are acknowledged for patient care and data acquisition. The authors acknowledge editorial assistance of Kim Coleman Healy and Jane M. Gilbert of Envision Scientific Solutions, which was contracted by Sanofi to provide publication support services.

\section{DISCLOSURE}

Z.B.K. received grant support from the Lysosomal Disease Network; Sanofi Genzyme. A.K.D. received grant support from Sanofi Genzyme. D.K. received research funding from Sanofi Genzyme and New York Medical College. S.P. is a recipient of a research grant from Sanofi Genzyme, member of Sanofi Genzyme speakers' bureau, and participant in a clinical trial of an unrelated product of Sanofi Genzyme. D.M.N. is a member of Sanofi Genzyme speakers' bureau. A.M.-W., C.S., A.M.J., and S.R. are employees of Sanofi Genzyme. P.S.K. has received research support and honoraria from and holds Pompe and Gaucher Disease Registries' advisory board membership at Sanofi Genzyme; has received grants and honoraria from Shire Pharmaceuticals, Valerion, Amicus; and Alexion Pharmaceuticals, Inc., and holds advisory board membership at Baebies, Inc. The other authors declare no conflicts of interest.

\section{REFERENCES}

1. Hirschhorn R, Reuser AJJ. In: Valle D, Beaudet AL, Vogelstein B, Kinzler K, Antonarakis SE, Ballabio A, Gibson KM, Mitchell G, eds. OMMBID: the online metabolic \& molecular bases of inherited disease. New York: McGraw-Hill. 2009

2. Kishnani PS, Hwu WL, Mandel H, Nicolino M, Yong F, Corzo D. A retrospective, multinational, multicenter study on the natural history of infantile-onset Pompe disease. J Pediatr. 2006;148:671-676.

3. van den Hout HM, Hop W, van Diggelen OP, et al. The natural course of infantile Pompe's disease: 20 original cases compared with 133 cases from the literature. Pediatrics. 2003;112:332-340.

4. Kishnani PS, Amartino HM, Lindberg $C$, et al. Timing of diagnosis of patients with Pompe disease: data from the Pompe registry. Am J Med Genet A. 2013;161A:2431-2443.

5. Genzyme Corporation. MYOZYME ${ }^{\circledR}$ (alglucosidase alfa) Prescribing information. https://www.myozyme.com/ /media/MyozymeUS/Files/ Documents/mz_pi.pdf. Accessed 23 June 2016. 
6. Kishnani PS, Corzo D, Nicolino M, et al. Recombinant human acid [alpha]glucosidase: major clinical benefits in infantile-onset Pompe disease. Neurology. 2007;68:99-109.

7. Kishnani PS, Nicolino M, Voit T, et al. Chinese hamster ovary cell-derived recombinant human acid alpha-glucosidase in infantile-onset Pompe disease. J Pediatr. 2006;149:89-97.

8. Nicolino M, Byrne B, Wraith JE, et al. Clinical outcomes after long-term treatment with alglucosidase alfa in infants and children with advanced Pompe disease. Genet Med. 2009;11:210-219.

9. Kishnani PS, Dickson PI, Muldowney L, et al. Immune response to enzyme replacement therapies in lysosomal storage diseases and the role of immune tolerance induction. Mol Genet Metab. 2016:117:66-83.

10. Kishnani PS, Goldenberg PC, DeArmey SL, et al. Cross-reactive immunologic material status affects treatment outcomes in Pompe disease infants. Mol Genet Metab. 2010;99:26-33.

11. Bali DS, Goldstein JL, Banugaria S, et al. Predicting cross-reactive immunological material (CRIM) status in Pompe disease using GAA mutations: lessons learned from 10 years of clinical laboratory testing experience. Am J Med Genet C Semin Med Genet. 2012; 160C:40-49

12. Berrier $\mathrm{KL}$, Kazi $Z \mathrm{~B}$, Prater $\mathrm{SN}$, et al. CRIM-negative infantile Pompe disease: characterization of immune responses in patients treated with ERT monotherapy. Genet Med. 2015;17:912-918.

13. Banugaria SG, Prater SN, Ng YK, et al. The impact of antibodies on clinical outcomes in diseases treated with therapeutic protein: lessons learned from infantile Pompe disease. Genet Med. 2011; 13:729-736.

14. Lacaná E, Yao LP, Pariser AR, Rosenberg AS. The role of immune tolerance induction in restoration of the efficacy of ERT in Pompe disease. Am J Med Genet C Semin Med Genet. 2012;160C:30-39.

15. Messinger $\mathrm{YH}$, Mendelsohn NJ, Rhead W, et al. Successful immune tolerance induction to enzyme replacement therapy in CRIM-negative infantile Pompe disease. Genet Med. 2012;14:135-142.

16. Elder ME, Nayak S, Collins SW, et al. B-cell depletion and immunomodulation before initiation of enzyme replacement therapy blocks the immune response to acid alpha-glucosidase in infantile-onset Pompe disease. J Pediatr. 2013;163:847-54 e841.

17. Mendelsohn NJ, Messinger YH, Rosenberg AS, Kishnani PS. Elimination of antibodies to recombinant enzyme in Pompe's disease. N Engl J Med. 2009;360:194-195.

18. Deodato F, Ginocchio VM, Onofri A, Grutter G, Germani A, Dionisi-Vici C. Immune tolerance induced using plasma exchange and rituximab in an infantile Pompe disease patient. J Child Neurol. 2014:29:850-854.

19. Hobson-Webb LD, Proia AD, Thurberg BL, Banugaria S, Prater SN, Kishnani PS. Autopsy findings in late-onset Pompe disease: a case report and systematic review of the literature. Mol Genet Metab. 2012;106:462-469.

20. Hunley TE, Corzo D, Dudek M, et al. Nephrotic syndrome complicating alpha-glucosidase replacement therapy for Pompe disease. Pediatrics. 2004:114:e532-e535

21. Banugaria SG, Prater SN, McGann JK, et al. Bortezomib in the rapid reduction of high sustained antibody titers in disorders treated with therapeutic protein: lessons learned from Pompe disease. Genet Med. 2013;15:123-131.
22. Banugaria SG, Patel TT, Mackey J, et al. Persistence of high sustained antibodies to enzyme replacement therapy despite extensive immunomodulatory therapy in an infant with Pompe disease: need for agents to target antibody-secreting plasma cells. Mol Genet Metab. 2012;105:677-680.

23. Banugaria SG, Prater SN, Patel TT, et al. Algorithm for the early diagnosis and treatment of patients with cross reactive immunologic materialnegative classic infantile pompe disease: a step towards improving the efficacy of ERT. PLoS ONE. 2013;8:e67052.

24. Kazi ZB, Desai AK, Berrier $K L$, et al. Sustained immune tolerance induction in enzyme replacement therapy-treated CRIM-negative patients with infantile Pompe disease. JCI Insight 2017;2. https://doi.org/10.1172/ jci.insight.94328

25. Stenger EO, Kazi Z, Lisi E, Gambello MJ, Kishnani P. Immune tolerance strategies in siblings with infantile Pompe disease-advantages for a preemptive approach to high-sustained antibody titers. Mol Genet Metab Rep. 2015;4:30-34

26. Genzyme Corporation. LUMIZYME (alglucosidase alfa) Prescribing information. August 2014. https://www.lumizyme.com/ /media/ LumizymeUS/Files/lumizyme_prescribing_information.pdf. Accessed 24 November 2016.

27. Joseph A, Munroe K, Housman M, Garman R, Richards S. Immune tolerance induction to enzyme-replacement therapy by co-administration of short-term, low-dose methotrexate in a murine Pompe disease model. Clin Exp Immunol. 2008;152:138-146.

28. Joly MS, Martin RP, Mitra-Kaushik S, et al. Transient low-dose methotrexate generates $B$ regulatory cells that mediate antigen-specific tolerance to alglucosidase alfa. J Immunol. 2014;193:3947-3958.

29. Mauri C, Menon M. The expanding family of regulatory B cells. Int Immunol. 2015:27:479-486.

30. Abbott MA, Prater SN, Banugaria SG, et al. Atypical immunologic response in a patient with CRIM-negative Pompe disease. Mol Genet Metab. 2011;104:583-586.

31. Kishnani PS, Corzo D, Nicolino M, et al. Recombinant human acid [alpha]glucosidase: major clinical benefits in infantile-onset Pompe disease. Neurology. 2007;68:99-109. (Erratum in Neurology. 2008 Nov 18;71 (21):1748).

32. Kazi ZB, Desai AK, Erwin A, et al. Prophylactic immune modulation in infantile Pompe disease using low-dose methotrexate induction: A safe, inexpensive, widely accessible, and efficacious strategy. Mol Genet Metab. 2016:117:S65-S66.

33. Garman RD, Munroe K, Richards SM. Methotrexate reduces antibody responses to recombinant human alpha-galactosidase $A$ therapy in a mouse model of Fabry disease. Clin Exp Immunol. 2004;137:496-502.

34. Sun B, Banugaria SG, Prater SN, et al. Non-depleting anti-CD4 monoclonal antibody induces immune tolerance to ERT in a murine model of Pompe disease. Mol Genet Metab Rep. 2014;1:446-450.

35. Cousens LP, Mingozzi F, van der Marel S, et al. Teaching tolerance: new approaches to enzyme replacement therapy for Pompe disease. Hum Vaccin Immunother. 2012:8:1459-1464.

36. Lim HH, Yi HQ, Kishimoto TK, Gao FQ, Sun BD, Kishnani PS. Immunomodulation to enzyme replacement therapy with tolerogenic nanoparticles containing rapamycin in a murine model of Pompe disease. Mol Genet Metab. 2017;120:S83-S84. 